\title{
Hard diffraction and Photon-induced processes with Proton Tag- ging at the LHC
}

\author{
Christophe Royon ${ }^{1, a}$ \\ ${ }^{1}$ Department of Physics and Astronomy 1082 Malott, 1251 Wescoe Hall Dr. Lawrence, KS 66045-758
}

\begin{abstract}
We describe some of the measurements that can be performed at the LHC by the TOTEM, CMS and ATLAS collaborations on hard diffraction in order to understand better the structure of the Pomeron. We will also describe the prospects concerning the search for quartic $\gamma \gamma \gamma \gamma$ anomalous couplings.
\end{abstract}

\section{The CMS-TOTEM and ATLAS detectors and their acceptance}

The TOTEM and ATLAS collaboration installed some roman pots located at about 200-220 meters from the CMS/ATLAS interaction point on both sides of CMS/ATLAS in order to measure intact protons after collisions [1]. In addition, there were some roman pot detectors located at $147 \mathrm{~m}$ but they were moved to $220 \mathrm{~m}$ during the 2015 shutdown.

It is possible to collect combined data between CMS and TOTEM [1]. The CMS-TOTEM collaboration aims at combining the data using the vertical roman pots of TOTEM and the CMS detector. The acceptance in $(\xi, t)$ for high $\beta^{*} \sim 90 \mathrm{~m}$ is given in Fig. 1, left $(t$ is the transfered energy squared at the proton vertex and $\xi$ is the proton momentum fraction carried by the Pomeron) and the acceptance for ATLAS is similar. We see that we have a good acceptance in a large domain in $\xi$ and thus in diffractive mass. The luminosity accumulated in special high- $\beta^{*}$ is rather low and the high- $\beta^{*}$ measurements are restricted to processes with a high enough cross sections. The CMS-TOTEM-Precision Proton Spectrometer (CT-PPS) and the ATLAS Forward Physics (AFP) detectors aim at taking data using the horizontal pots of TOTEM/AFP and the CMS/ATLAS detectors at high luminosity for the standard low- $\beta^{*}$ lattice parameters of the LHC. The acceptance is shown in Fig. 1, right. We see that we have a good acceptance for rather high values of $\xi$ up to 0.15 , so at high diffractive masses. For these runs, the luminosity is quite high and we are sensitive to processes with low cross section. The situation is thus complementary to the high- $\beta^{*}$ running scenario.

\section{Measurements at medium luminosities: the Pomeron structure}

\subsection{Mesaurement at medium luminosities: Hard diffraction}

In this section, we will discuss briefly how we can assess the gluon and quark contents in the Pomeron at the LHC, and also the search for BFKL resummation effects.

\footnotetext{
a e-mail: christophe.royon@ku.edu
} 

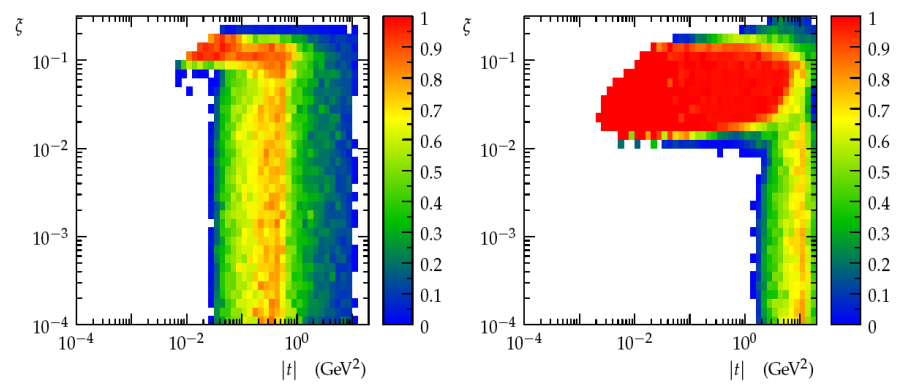

Figure 1. Acceptance in $(t, \xi)$ of the roman pots detectors for high- $\beta^{*}$ (left) and low- $\beta^{*}$ (right).
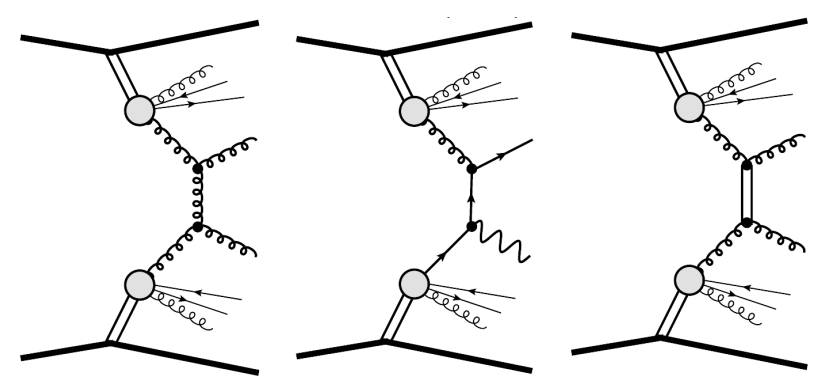

Figure 2. Inclusive diffractive diagrams. From left to right: jet production in inclusive double pomeron exchange, $\gamma+$ jet production in DPE, jet gap jet events

Inclusive diffraction measurements at the LHC We discuss potential measurements at the LHC that can constrain the Pomeron structure. The Pomeron structure in terms of quarks and gluons has been derived from QCD fits at HERA and it is possible to probe this structure and the QCD evolution at the LHC in a completely new kinematical domain. All the following studies have been performed using the Forward Physics Monte Carlo (FPMC), a generator that has been designed to study forward physics, especially at the LHC [3]. It aims to provide a variety of diffractive processes in one common framework, i.e. single diffraction, double pomeron exchange, central exclusive production and twophoton exchange.

\section{Dijet production in double Pomeron exchanges processes and sensitivity to the gluon density in the pomeron}

One can first probe if the Pomeron is universal between $e p$ and $p p$ colliders, or in other words, if we are sensitive to the same object at HERA and the LHC using as an example dijet production in single diffractive and double pomeron exchange at the LHC. It is possible to assess the gluon and quark densities using the dijet and $\gamma+$ jet productions. The different diagrams of the processes that can be studied at the LHC are shown in Fig. 2, namely double pomeron exchange (DPE) production of dijets (left), of $\gamma+$ jet (middle), sensitive respectively to the gluon and quark contents of the Pomeron, and the jet gap jet events (right).

The dijet production in DPE events at the LHC is sensitive to the gluon density in the Pomeron. In order to quantify how well we are sensitive to the Pomeron structure in terms of gluon density at 


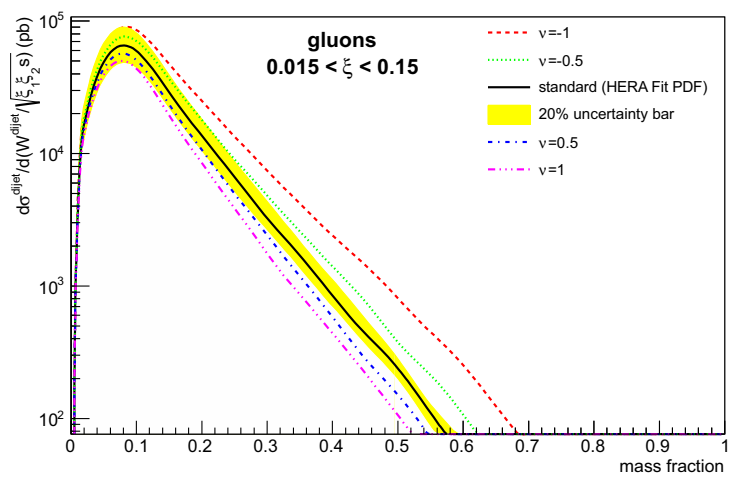

Figure 3. DPE di-jet mass fraction distribution. The different curves correspond to different modifications of the Pomeron gluon density extracted from HERA data (see text).

the LHC, we display in Fig. 3, the dijet mass fraction, the ratio of the dijet mass to the total diffractive mass $[4,5]$. The central black line displays the cross section value for the gluon density in the Pomeron measured at HERA including an additional survival probability of 0.03 . The yellow band shows the effect of the $20 \%$ uncertainty on the gluon density taking into account the normalisation uncertainties. The dashed curves display how the dijet cross section at the LHC is sensitive to the gluon density distribution especially at high $\beta$. For this sake, we multiply the gluon density in the Pomeron from HERA by $(1-\beta)^{v}$ where $v$ varies between -1 and 1 . When $v$ is equal to -1 (resp. 1), the gluon density is enhanced (resp, decreased) at high $\beta$. We note that the curves corresponding to the different values of $v$ are much more separated at high values of the dijet mass fraction, meaning that this observable is indeed sensitive to the gluon density at high $\beta$.

\section{Sensitivity to the Pomeron structure in quarks using $\gamma+$ jet events and $W$ asymmetry}

The QCD diffractive fits performed at HERA assumed that $u=d=s=\bar{u}=\bar{d}=\bar{s}$, since data were not sensitive to the difference between the different quark component in the Pomeron. On the contrary, measuring the $\gamma+$ jet to the dijet cross section ratios as a function of the diffractive mass $M$ allows to distinguish between different assumptions on the quark content of the Pomeron. For instance, varying $d / u$ between 0.25 and 4 leads to a variation of the cross section ratio by a factor 2.5 .

In addition, it is possible to use the $W$ asymmetry in single diffractive $W$ production [6]. Typically, the muon asymmetry is directly sensitive to the quark content of the pomeron and varies by a factor 6 at low $\xi$ between the assumptions $u / d=2$ or $u / d=1 / 2$ for the quark content in the pomeron.

Reggeon contribution at high $\xi$ The dijet diffractive cross section is also sensitive to Reggeon exchanges especially at high $\xi$. The Reggeon contribution is porrly constrained at HERA and it is important to measure it at the LHC. In Ref. [9], we showed that the Reggeon contribution is of the same order of magnitude as the Pomeron one in single diffractive dijets for $0.1<\xi<0.17$.

\section{Jet gap jet production in double Pomeron exchanges processes}

The observation of gap between jets in double Pomeron exchange events [8, 10] is ideal to probe the BFKL [7] evolution equation. We predict that the ratio of jet-gap-jet with respect with "standard" dijet production in DPE events will be of the order of $20 \%$, which would represented a good test of the BFKL evolution equation. 


\subsection{Exclusive processes in diffraction}

The advantage of the exclusive diffractive and photon exchange processes is that all particles can be measured in the final state. Both protons can be measured in CMS-TOTEM/CT-PPS and the produced particles (jets, vector mesons, $Z$ boson....) in CMS/ATLAS, and there is no energy losses such as in the pomeron remnants. It is thus possible to reconstruct the properties of the object produced exclusively (via photon and gluon exchanges) from the tagged proton since the system is completely constrained. It is worth mentioning that it is also possible to constrain the background by requesting the matching between the information of the two protons and the produced object, and thus, central exclusive production is a potential channel for beyond standard model physics at high masses.

For instance, the CMS-TOTEM experiment performed extensive studies of possible measurements of exclusive states at high $\beta^{*}$. It is worth mentioning that the search for glueball states and the probe of the low $x$ gluon density down to $x \sim 10^{-4}$ will be possible. It is possible to discover or exclude glueballs at low masses since the $1-10 \mathrm{GeV}$ region in masses can be probed diffractively $\left(\xi \sim 10^{-4}-\right.$ $10^{-3}$ ), ensuring pure gluonic exchanges. It is then possible to check the $f_{0}(1500)$ or $f_{0}(1710)$ glueball candidates (lattice calculations predict a $0++$ glueball at $1.7 \mathrm{GeV}$ with a $\sim 100 \mathrm{MeV}$ uncertainty, favoring the $f_{0}(1710)$ candidate). The simulation of a possible signal of $f_{0}(1710) \rightarrow \rho^{0} \rho^{0}$ and of the non resonant $\rho^{0} \rho^{0}$ background including the CMS tracker performance leading to a $20-30 \mathrm{MeV}$ resolution was performed and a luminosity of $\sim 0.06 \mathrm{pb}^{-1}$ is needed for a $7 \sigma$ signal. About 0.6 $\mathrm{pb}^{-1}$ is needed for decay characterisation In addition, it is possible to perform a spin analysis of $f_{0}(1710) \rightarrow \rho^{0} \rho^{0} \rightarrow 4 \pi$ to determine if $J=0$ or 2 : as an example, a measurement of the polar angle of the $\pi^{+} \pi^{-}$pair for the $\rho$ candidate needs about $5 \mathrm{pb}^{-1}$

In addition, using the same data set, the measurement of the cross section times branching ratio for the three $\chi_{C, 0,1,2}$ states will be performed allowing a comparison with the results of the $\mathrm{LHCb}$ measurement.

\section{Search for quartic $\gamma \gamma \gamma \gamma$ anomalous coupling events in diffraction}

In this section, we will describe briefly the search for $\gamma \gamma \gamma \gamma$ quartic anomalous couplings using proton tagging at the LHC that regained interest after the excess observed in diphoton production by the CMS and ATLAS experiments [11, 12] (the search for $W W \gamma \gamma$ and $Z Z \gamma \gamma$ couplings is given in [13]).

\subsection{Theoretical motivations}

Four-photon $(4 \gamma)$ interactions through diphoton production via photon fusion with intact outgoing protons are considered. The pure photon dimension-eight operators read

$$
\mathcal{L}_{4 \gamma}=\zeta_{1}^{\gamma} F_{\mu \nu} F^{\mu \nu} F_{\rho \sigma} F^{\rho \sigma}+\zeta_{2}^{\gamma} F_{\mu \nu} F^{\nu \rho} F_{\rho \lambda} F^{\lambda \mu}
$$

and they can induce the $\gamma \gamma \gamma \gamma$ process, highly suppressed in the SM [14]. We discuss here possible new physics contributions to $\zeta_{1,2}^{\gamma}$ that can be probed and discovered at the LHC using the forward proton detectors.

Loops of heavy charged particles contribute to the $4 \gamma$ couplings [14] as $\zeta_{i}^{\gamma}=\alpha_{\mathrm{em}}^{2} Q^{4} m^{-4} N c_{i, s}$, where $c_{1, s}$ is related to the spin of the heavy particle of mass $m$ running in the loop and $Q$ its electric charge. These couplings scale as $\sim Q^{4}$ and are enhanced in presence of particles with large charges. For a $500 \mathrm{GeV}$ vector (fermion) resonance with $Q=3(4)$, large couplings $\zeta_{i}^{\gamma}$ of the order of $10^{-13}-$ $10^{-14} \mathrm{Gev}^{-4}$ can be reached.

Beyond perturbative contributions to $\zeta_{i}^{\gamma}$ from charged particles, non-renor- malizable interactions of neutral particles are also present in common extensions of the SM. Such theories can contain scalar, 
Table 1. Number of signal for $Q_{\text {eff }}=4, m=340 \mathrm{GeV}$ and background events after various selections for an integrated luminosity of $300 \mathrm{fb}^{-1}$ and $\mu=50$ at $\sqrt{s}=14 \mathrm{TeV}$. Values obtained using the corresponding EFT couplings with and without form factors are also displayed. Excl. stands for exclusive backgrounds and DPE for double pomeron exchange backgrounds.

\begin{tabular}{|c||c|c||c|c|c|c|}
\hline Cut / Process & $\begin{array}{c}\text { Signal } \\
\text { (full) }\end{array}$ & $\begin{array}{c}\text { Signal } \\
\text { with/without } \\
\text { f.f. (EFT) }\end{array}$ & Excl. & DPE & $\begin{array}{c}\text { DY } \\
\text { dijet+ }+ \\
\text { pile up }\end{array}$ & $\begin{array}{c}\gamma \gamma+ \\
\text { pile up }\end{array}$ \\
\hline \hline $\begin{array}{c}{\left[0.015<\xi_{1,2}<0.15,\right.} \\
p_{\mathrm{T} 1,(2)}>200\end{array}$ & 65. & $18 .(187)$. & 0.13 & 0.2 & 1.6 & 2968 \\
$\begin{array}{c}(100) \mathrm{GeV}] \\
m_{\gamma \gamma}>600 \mathrm{GeV}\end{array}$ & 64. & $17 .(186)$. & 0.10 & 0 & 0.2 & 1023 \\
{$\left[p_{\mathrm{T} 2} / p_{\mathrm{T} 1}>0.95\right.$,} & 64. & $17 .(186)$. & 0.10 & 0 & 0 & 80.2 \\
$|\Delta \phi|>\pi-0.01]$ & 61. & $12 .(175)$. & 0.09 & 0 & 0 & 2.8 \\
$\sqrt{\xi_{1} \xi_{2} s}=m_{\gamma \gamma} \pm 3 \%$ & 60. & $16 .(169)$. & 0.09 & 0 & 0 & 0 \\
$\left|y_{\gamma \gamma}-y_{p p}\right|<0.03$ & 60.0 & & & & \\
\hline
\end{tabular}

pseudo-scalar and spin-2 resonances that couple to the photon and generate the $4 \gamma$ couplings by treelevel exchange as $\zeta_{i}^{\gamma}=\left(f_{s} m\right)^{-2} d_{i, s}$, where $d_{1, s}$ is related to the spin of the particle.

\subsection{Experimental sensitivity to quartic four photon couplings}

The $\gamma \gamma \gamma \gamma$ process can be probed via the detection of two intact protons in the forward proton detectors and two energetic photons in the corresponding electromagnetic calorimeters.

The anomalous $\gamma \gamma \gamma \gamma$ process has been implemented in the Forward Physics Monte Carlo (FPMC) generator [3]. The FPMC generator was also used to simulate the background processes giving rise to two intact protons accompanied by two photons, electrons or jets that can mimic the photon signal. Those include exclusive SM production of $\gamma \gamma \gamma \gamma$ via lepton and quark boxes and $\gamma \gamma \rightarrow e^{+} e^{-}$. The central exclusive production of $\gamma \gamma$ via two-gluon exchange, was simulated using ExHuME. This series of backgrounds is called "Exclusive" in Table 1. FPMC was also used to produce $\gamma \gamma$, Higgs to $\gamma \gamma$ and dijet productions via double pomeron exchange (called DPE background in Table 1 and Fig. 4). Such backgrounds tend to be softer than the signal and can be suppressed with requirements on the transverse momenta of the photons and the diphoton invariant mass. In addition, the final-state photons of the signal are typically back-to-back and have about the same transverse momenta. Requiring a large azimuthal angle $|\Delta \phi|>\pi-0.01$ between the two photons and a ratio $p_{T, 2} / p_{T, 1}>0.95$ greatly reduces the contribution of non-exclusive processes.

Additional background processes include the quark and gluon-initiated production of two photons, two jets and Drell-Yan processes leading to two electrons. The two intact protons arise from pile-up interactions (these backgrounds are called $\gamma \gamma+$ pile-up and DY, dijet + pile-up in Table 1). The pile-up background is further suppressed by requiring the proton missing invariant mass to match the diphoton invariant mass within the expected resolution and the diphoton system rapidity and the rapidity of the two protons to be similar.

The number of expected signal and background events passing respective selections is shown in Table 1 for an integrated luminosity of $300 \mathrm{fb}^{-1}$ for a center-of-mass energy of $14 \mathrm{TeV}$. Exploiting the full event kinematics with the forward proton detectors allows to completely suppress the background with a signal selection efficiency after the acceptance cuts exceeding $70 \%$. Tagging the protons is absolutely needed to suppress the $\gamma \gamma+$ pile-up events. Further background reduction is even possible 

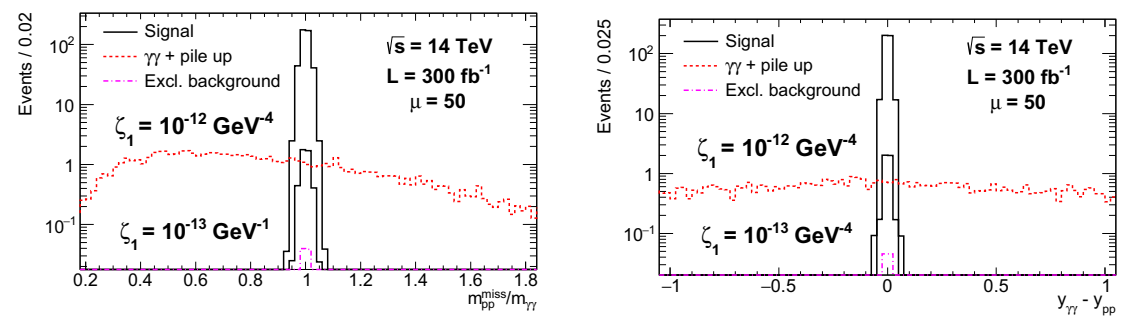

Figure 4. Diphoton to missing proton mass ratio (left) and rapidity difference (right) distributions for signal and backgrounds.

by requiring the photons and the protons to originate from the same vertex that provides an additional rejection factor of 40 for 50 pile-up interactions, showing the large margin on the background suppression. A similar study at a higher pile-up of 200 was performed and led to a very small background. The sensitivity extends up to $7 \cdot 10^{-15} \mathrm{GeV}^{-4}$ allowing us to probe further the models of new physics described above.

We also performed a full amplitude calculation in Ref. [11, 14] that avoids the dependence on the choice of form factors needed in order to avoid quadratic divergences of scattering amplitudes. Sensitivities were found to be similar leading to possible discoveries of vector or fermions at high masses and high effective charges.

In this short report, we presented present and potential measurements to be performed by the TOTEM and CMS collaborations related to a better understanding of the pomeron structure in terms of QCD and to exploratory physics where unprecedented sensitivities can be reached on quartic anomalous $\gamma \gamma \gamma \gamma$ couplings (as well as for $\gamma \gamma W W$ and $\gamma \gamma Z Z$ anomalous couplings).

\section{Acknowledgments}

The author thanks the organizers for partial support.

\section{References}

[1] TOTEM Coll., CERN-LHCC-2014- 020; CMS and TOTEM Coll., CERN-LHCC-2014-021; ATLAS Coll., CERN-LHCC-2011-012.

[2] TOTEM Coll., Europhysics Lett. 101 (2013) 21004; Europhysics Lett. 101 (2013) 21003; Europhysics Lett. 101 (2013) 21002; Europhysics Lett. 96 (2011) 21002; Europhysics Lett. 95 (2011) 41001; Nucl. Phys. B 899 (2015) 527; Phys. Rev. Lett. 111, 012001 (2013).

[3] M. Boonekamp et al., e-Print: arXiv:1102.2531;

[4] C, Marquet, C. Royon, M. Saimpert, D. Werder, Phys.Rev. D 88 (2013) 7, 074029.

[5] O. Kepka, C. Royon, Phys.Rev. D76 (2007) 034012; CDF Coll., Phys. Rev. D 77, (2008) 052004.

[6] A. Chuinard, C. Royon, Rafal Staszewski, preprint arXiv:1510.04218.

[7] V. S. Fadin, E. A. Kuraev, L. N. Lipatov, Phys. Lett. B60 (1975) 50; I. I. Balitsky, L. N. Lipatov, Sov.J.Nucl.Phys. 28 (1978) 822;

[8] C. Marquet, C. Royon, M. Trzebinski, R. Zlebcik, Phys.Rev. D87 (2013) 3, 034010; O. Kepka, C. Marquet, C. Royon, Phys. Rev. D83 (2011) 034036. 
[9] C. Marquet, D.E. Martins, A.V. Pereira, M. Rangel, C. Royon, preprint arXiv:1608.05674.

[10] C. Marquet, C. Royon, Phys. Rev. D79 (2009) 034028; O. Kepka, C. Royon, C. Marquet, R. Peschanski, Eur. Phys .J. C55 (2008) 259-272; Phys. Lett. B655 (2007) 236-240; H. Navelet, R. Peschanski, C. Royon, S. Wallon, Phys. Lett. B385 (1996) 357; H. Navelet, R. Peschanski, C. Royon, Phys. Lett. B366 (1996) 329.

[11] S. Fichet, G. von Gersdorff, C. Royon, preprint : arXiv:1512.05751; S. Fichet, G. von Gersdorff, C. Royon, preprint : arXiv:1601.01712.

[12] CMS Coll., CMS-PAS-EXO-15-004; ATLAS Coll., ATLAS-CONF-2015-081.

[13] E. Chapon, O. Kepka, C. Royon, Phys. Rev. D81 (2010) 074003; O. Kepka and C. Royon, Phys. Rev. D 78 (2008) 073005.

[14] S. Fichet, G. von Gersdorff, B. Lenzi, C. Royon, M. Saimpert, JHEP 1502 (2015) 165; S. Fichet, G. von Gersdorff, O. Kepka, B. Lenzi, C. Royon, M. Saimpert, Phys.Rev. D89 (2014) 114004.

[15] S. Fichet, G. von Gersdorff, C. Royon, Phys.Rev.Lett. 116 (2016) no.23, 231801; S. Fichet, G. von Gersdorff, C. Royon, Phys.Rev. D93 (2016) no.7, 075031. 